\title{
Semiclassical description of relativistic spin without use of Grassmann variables and the Dirac equation
}

\author{
A. A. Deriglazov* \\ Dept. de Matematica, ICE, Universidade Federal de Juiz de Fora, \\ MG, Brazil.
}

\begin{abstract}
We propose a relativistic particle model without Grassmann variables which, being canonically quantized, leads to the Dirac equation. Both $\Gamma$ matrices and the relativistic spin tensor are produced through the canonical quantization of the classical variables which parametrize the properly constructed relativistic spin surface. Although there is no mass-shell constraint in our model, our particle's speed cannot exceed the speed of light. The classical dynamics of the model is in correspondence with the dynamics of mean values of the corresponding operators in the Dirac theory. In particular, the position variable experiences Zitterbewegung in noninteracting theory. The classical equations for the spin tensor are the same as those of the Barut-Zanghi model of a spinning particle.
\end{abstract}

\section{Introduction}

Starting from the classical works [1-4], a lot of effort has been spent on attempts to understand the behavior of a particle with spin on the basis of semiclassical mechanical models [5-16, 21-27]. One possibility is to use the Grassmann (anticommutative) variables for parametrization of the spin space $[11,12]$. In their pioneer work [11], Berezin and Marinov have suggested such a kind model. Their prescription can be briefly summarized as follows. For nonrelativistic spin, the noninteracting Lagrangian reads $\frac{m}{2}\left(\dot{x}_{i}\right)^{2}+\frac{i}{2} \xi_{i} \dot{\xi}_{i}$, where the spin inner space is constructed from vector-like Grassmann variables $\xi_{i}, \xi_{i} \xi_{j}=-\xi_{j} \xi_{i}$. Since the Lagrangian is linear on $\dot{\xi}_{i}$, their conjugate

*alexei.deriglazov@ufjf.edu.br On leave of absence from Dept. Math. Phys., Tomsk Polytechnical University, Tomsk, Russia. 
momenta coincide with $\xi, \pi_{i}=\frac{\partial L}{\partial \dot{\xi}_{i}}=i \xi_{i}$. The relations represent the Dirac second-class constraints and are taken into account by the transition from the Poisson (Grassmann) bracket to the Dirac one, the latter reading

$$
\left\{\xi_{i}, \xi_{j}\right\}_{D B}=i \delta_{i j}
$$

Dealing with the Dirac bracket, one can resolve the constraints, excluding the momenta from consideration. This gives a very economic scheme for the description of a spin: there are only three spin variables $\xi_{i}$ with the desired brackets (11). Canonical quantization is performed, replacing the variables by the spin operators proportional to the Pauli $\sigma$-matrices, $\hat{S}_{i}=\frac{\hbar}{2} \sigma^{i}\left(\left[\sigma^{i}, \sigma^{j}\right]_{+}=2 \delta^{i j}\right)$ :

$$
\left[\hat{S}_{i}, \hat{S}_{j}\right]_{+}=\frac{\hbar^{2}}{2} \delta_{i j}
$$

They act on the two-dimensional spinor space $\Psi_{\alpha}$. Canonical quantization of the particle on an external electromagnetic background leads to the Pauli equation

$$
i \hbar \frac{\partial \Psi}{\partial t}=\left(\frac{1}{2 m}\left(\hat{\mathbf{p}}-\frac{e}{c} \mathbf{A}\right)^{2}-e A_{0}-\frac{e \hbar}{2 m c} \boldsymbol{\sigma} \mathbf{B}\right) \Psi .
$$

Relativistic spin is described in a similar way $[11,12]$.

The problem here is that the Grassmann classical mechanics represents a rather formal mathematical construction. It leads to certain difficulties $[11,16]$ in attempts to use it for describing the spin effects on the semiclassical level, before the quantization. Hence it would be interesting to describe spin on the basis of usual variables. While the problem has a long history (see [5-13] and references therein), there appears to be no wholly satisfactory solution to date. It seems to be surprisingly difficult [13] to construct, in a systematic way, a consistent model that would lead to the Dirac equation in the the course of canonical quantization. It is the aim of this work to construct an example of a mechanical model for the one-particle sector of the Dirac equation.

To describe the nonrelativistic spin using commuting variables, we need to realize the commutator algebra of the operators $\hat{S}_{i}$

$$
\left[\hat{S}_{i}, \hat{S}_{j}\right]_{-}=i \hbar \epsilon_{i j k} \hat{S}_{k}
$$


instead of the anticommutator one (2). This has been achieved in the recent work [15] starting from the Lagrangian

$$
\begin{gathered}
S=\int d t\left(\frac{m}{2}\left(\dot{x}_{i}\right)^{2}+\frac{e}{c} A_{i} \dot{x}_{i}+e A_{0}\right. \\
\left.+\frac{1}{2 g}\left(\dot{\omega}_{i}-\frac{e}{m c} \epsilon_{i j k} \omega_{j} B_{k}\right)^{2}+\frac{3 g \hbar^{2}}{8 a^{2}}+\frac{1}{\phi}\left(\left(\omega_{i}\right)^{2}-a^{2}\right)\right) .
\end{gathered}
$$

The configuration-space variables are $x_{i}(t), \omega_{i}(t), g(t)$ and $\phi(t)$. Here $x_{i}$ represents the spatial coordinates of the particle with the mass $m$ and the charge $e, \omega_{i}$ are the spin-space coordinates, $g, \phi$ are the auxiliary variables, $a=$ const and $\mathbf{B}=\boldsymbol{\nabla} \times \mathbf{A}$. The second and third terms in Eq. (15) represent minimal interaction with the vector potential $A_{0}, A_{i}$ of an external electromagnetic field, while the fourth term contains the interaction of the spin with a magnetic field. At the end, it produces the Pauli term in the quantum mechanical Hamiltonian.

The Dirac constraints presented in the model imply [15] that spin lives on a two-dimensional surface of a six-dimensional spin phase space $\omega_{i}, \pi_{i}$. The surface can be parametrized with the angularmomentum coordinates $S_{i}=\epsilon_{i j k} \omega_{j} \pi_{k}$, subject to $S^{2}=\frac{3 \hbar^{2}}{4}$. They obey the classical brackets $\left\{S_{i}, S_{j}\right\}=\epsilon_{i j k} S_{k}$. Hence we quantize them according to the rule $S_{i} \rightarrow \hat{S}_{i}$.

The action leads to a reasonable picture on both classical and quantum levels. The classical dynamics is governed by the Lagrangian equations

$$
\begin{gathered}
m \ddot{x}_{i}=e E_{i}+\frac{e}{c} \epsilon_{i j k} \dot{x}_{j} B_{k}-\frac{e}{m c} S_{k} \partial_{i} B_{k}, \\
\dot{S}_{i}=\frac{e}{m c} \epsilon_{i j k} S_{j} B_{k} .
\end{gathered}
$$

We have defined $\mathbf{E}=-\frac{1}{c} \frac{\partial \mathbf{A}}{\partial t}+\nabla A_{0}$. Since $S^{2} \approx \hbar^{2}$, the $S$-term disappears from Eq. (6) in the classical limit $\hbar \rightarrow 0$. Then Eq. (6) reproduces the classical motion on an external electromagnetic field. Notice also that in the absence of interaction, the spinning particle does not experience an undesirable Zitterbewegung. Equation (17) describes the classical spin precession in an external magnetic field. On the other hand, canonical quantization of the model immediately produces the Pauli equation (3). 
Below, we generalize this scheme to the relativistic case, taking angular-momentum variables as the basic coordinates of the spin space. On this basis we construct the relativistically invariant classical mechanics that produces the Dirac equation through the canonical quantization, and briefly discuss its classical dynamics.

\section{Relativistic spin surface}

Here we motivate our choice of the variables for describing the relativistic spin. The dynamical model based on these variables is constructed in the next section. The relativistic equation for the spin precession can be obtained including the three-dimensional spin vector $S_{i}$ (4) either into the Frenkel tensor $\Phi^{\mu \nu}, \Phi^{\mu \nu} u_{\nu}=0$, or into the Bargmann-Michel-Telegdi 4-vector $S^{\mu}, S^{\mu} u_{\mu}=0$, where $u_{\mu}$ is the 4 -velocity vector 1 . Unfortunately, the semiclassical models based on these schemes do not lead to a reasonable quantum theory, as they do not produce the Dirac equation through the canonical quantization. We now motivate as to how this can be achieved in the formulation that implies the inclusion of $S_{i}$ into the $S O(2,3)$ angular-momentum tensor $J^{A B}$ of five-dimensional space $A=(\mu, 5)=(0,1,2,3,5), \eta^{A B}=(-+++-)$.

In the passage from nonrelativistic to relativistic spin, we replace the Pauli equation by the Dirac one:

$$
\left(\hat{p}_{\mu} \Gamma^{\mu}+m c\right) \Psi\left(x^{\mu}\right)=0,
$$

where $\hat{p}_{\mu}=-i \hbar \partial_{\mu}$. The position of the particle is described in the standard way; the corresponding phase-space variables are $x^{\mu}, p^{\nu}$, $\left\{x^{\mu}, p^{\nu}\right\}_{P B}=\eta^{\mu \nu}, \eta^{\mu \nu}=(-+++)$.

Let us look for the classical variables that could produce the $\Gamma$-matrices. According to the canonical quantization paradigm, the classical variables, say $z^{\alpha}$, corresponding to the Hermitian operators $\hat{z}^{\alpha}$ must be chosen to obey the quantization rule

$$
\left[\hat{z}^{\alpha}, \hat{z}^{\beta}\right]_{-}=\left.i \hbar\left\{z^{\alpha}, z^{\beta}\right\}\right|_{z \rightarrow \hat{z}} .
$$

In this equation, $[,]_{-}$is the commutator of the operators and $\{$, stands for the classical bracket 2 . To avoid the operator-ordering

\footnotetext{
${ }^{1}$ The conditions $\Phi^{\mu \nu} u_{\nu}=0$ and $S^{\mu} u_{\mu}=0$ guarantee that in the rest frame, only three components of these quantities survive, which implies the right nonrelativistic limit.

${ }^{2}$ It is the Poisson (Dirac) bracket in a theory without (with) second-class constraints.
} 
problems, we will consider only the sets of operators which form the Lie algebra, $\left[\hat{z}^{\alpha}, \hat{z}^{\beta}\right]_{-}=c^{\alpha \beta}{ }_{\gamma} \hat{z}^{\gamma}$. So our first task is to study the algebra of $\Gamma$-matrices. We note that commutators of $\Gamma^{\mu}$ do not form closed Lie algebra, but produce $S O(1,3)$ Lorentz generators

$$
\left[\Gamma^{\mu}, \Gamma^{\nu}\right]_{-}=-2 i \Gamma^{\mu \nu},
$$

where $\Gamma^{\mu \nu} \equiv \frac{i}{2}\left(\Gamma^{\mu} \Gamma^{\nu}-\Gamma^{\nu} \Gamma^{\mu}\right)$. The set $\Gamma^{\mu}, \Gamma^{\mu \nu}$ forms a closed algebra. Besides the commutator (10), we have

$$
\begin{gathered}
{\left[\Gamma^{\mu \nu}, \Gamma^{\alpha}\right]_{-}=2 i\left(\eta^{\mu \alpha} \Gamma^{\nu}-\eta^{\nu \alpha} \Gamma^{\mu}\right),} \\
{\left[\Gamma^{\mu \nu}, \Gamma^{\alpha \beta}\right]_{-}=2 i\left(\eta^{\mu \alpha} \Gamma^{\nu \beta}-\eta^{\mu \beta} \Gamma^{\nu \alpha}-\eta^{\nu \alpha} \Gamma^{\mu \beta}+\eta^{\nu \beta} \Gamma^{\mu \alpha}\right) .}
\end{gathered}
$$

The algebra can be identified with $S O(2,3)$ Lorentz algebra with generators $\hat{L}^{A B}$ :

$$
\left[\hat{L}^{A B}, \hat{L}^{C D}\right]_{-}=2 i\left(\eta^{A C} \hat{L}^{B D}-\eta^{A D} \hat{L}^{B C}-\eta^{B C} \hat{L}^{A D}+\eta^{B D} \hat{L}^{A C}\right),
$$

assuming that $\Gamma^{\mu} \equiv \hat{L}^{5 \mu}, \Gamma^{\mu \nu} \equiv \hat{L}^{\mu \nu}$.

According to Eqs. (9) and (12) we need classical variables with the algebra

$$
\left\{J^{A B}, J^{C D}\right\}=2\left(\eta^{A C} J^{B D}-\eta^{A D} J^{B C}-\eta^{B C} J^{A D}+\eta^{B D} J^{A C}\right) .
$$

The problem is that in classical mechanics the basic phase-space variables, say $\omega^{\alpha}, \pi_{\beta}$, necessarily obey the Poisson bracket $\left\{\omega^{\alpha}, \pi_{\beta}\right\}=$ $\delta^{\alpha}{ }_{\beta}$. The algebra differs from (13). So we generally need to pass from the initial to some composed variables as well as to impose some constraints. This implies the use of the Dirac machinery for constrained theories $[16,17]$.

To arrive at the algebra (13), we introduce, tentatively, the tendimensional "phase" space of the spin degrees of freedom, $\omega^{A}, \pi^{B}$, equipped with the Poisson bracket

$$
\left\{\omega^{A}, \pi^{B}\right\}_{P B}=\eta^{A B},
$$

and define the inner angular momentum

$$
J^{A B} \equiv 2\left(\omega^{A} \pi^{B}-\omega^{B} \pi^{A}\right) .
$$

The Poisson brackets of these quantities form the desired classical algebra (13).

Below we use the decompositions $(i, j=1,2,3)$

$$
J^{A B}=\left(J^{5 \mu}, J^{\mu \nu}\right)=\left(J^{50}, J^{5 i}=\mathbf{J}^{5}, J^{0 i}=\mathbf{W}, J^{i j}=\epsilon^{i j k} D^{k}\right) .
$$


Since the $J^{A B}$ are the variables which we are interested in, we try to take them as coordinates of the space $\omega^{A}, \pi^{B}$. The Jacobian of the transformation $\left(\omega^{A}, \pi^{B}\right) \rightarrow J^{A B}$ has rank equal to 7 . So, only seven among the ten functions $J^{A B}(\omega, \pi), A<B$, are independent quantities. They can be separated as follows. By construction, they obey the identity

$$
\epsilon^{\mu \nu \alpha \beta} J^{5}{ }_{\nu} J_{\alpha \beta}=0, \Leftrightarrow J^{i j}=\left(J^{50}\right)^{-1}\left(J^{5 i} J^{0 j}-J^{5 j} J^{0 i}\right),
$$

that is the 3 -vector $\mathbf{D}$ can be presented through $\mathbf{J}^{5}, \mathbf{W}$

$$
\mathbf{D}=\frac{1}{J^{50}} \mathbf{J}^{5} \times \mathbf{W}
$$

Hence we can take $J^{5 \mu}$ and $J^{0 i}$ as a part of the new coordinate system. Suppose, we complete this set up to a basis of the phase space adding three more coordinates, say $a, b, c$. Quantizing the complete set we obtain, besides the desired operators $\hat{L}^{5 \mu}, \hat{L}^{0 i}$, some extra operators $\hat{a}, \hat{b}, \hat{c}$. They are not present in the Dirac theory, and are not necessary for the description of the spin. So we need to reduce the dimension of our space from 10 to 7 , imposing three constraints. There is one important restriction on the choice of constraints. Canonical quantization of a system with constraints implies replacement of the Poisson by the Dirac bracket; the latter is constructed with the help of the constraints. We need $S O(2,3)$ invariant constraints $T_{a},\left\{T_{a}, J^{A B}\right\}_{P B}=0$; otherwise the Diracbracket algebra will not coincide with those of the Poisson, (13).

The only quadratic $S O(2,3)$-invariants which can be constructed from $\omega^{A}, \pi^{B}$ are $\omega^{A} \omega_{A}, \omega^{A} \pi_{A}$ and $\pi^{A} \pi_{A}$. So we restrict our model to living on the surface defined by the equations

$$
\begin{gathered}
T_{3}=\pi^{A} \pi_{A}+a_{3}=0 \\
T_{4}=\omega^{A} \omega_{A}+a_{4}=0, \quad T_{5}=\omega^{A} \pi_{A}=0,
\end{gathered}
$$

where $a_{3}, a_{4}$ are some numbers. Our suggestion is to take the surface as the inner space for the description of the relativistic spin.

The matrix $\frac{\partial\left(J^{5 \mu}, J^{0 i}, T_{4}, T_{5}, \omega^{5}\right)}{\partial\left(\omega^{A}, \pi^{B}\right)}$ has rank equal ten. So the quantities

$$
J^{5 \mu}, J^{0 i}, T_{4}, T_{5}, \omega^{5},
$$


can be taken as coordinates of the space $\left(\omega^{A}, \pi^{B}\right)$. The equation $J^{A B}=2\left(\omega^{A} \pi^{B}-\omega^{B} \pi^{A}\right)$ implies the identity

$$
\begin{gathered}
J^{A B} J_{A B}=8\left[\left(\omega^{A}\right)^{2}\left(\pi^{B}\right)^{2}-\left(\omega^{A} \pi_{A}\right)^{2}\right]= \\
8\left[\left(T_{4}-a_{4}\right)\left(T_{3}-a_{3}\right)-\left(T_{5}\right)^{2}\right],
\end{gathered}
$$

then the constraint $T_{3}$ can be written in the coordinates (21) as follows:

$$
T_{3}=\frac{\left(J^{A B}\right)^{2}+8\left(T_{5}\right)^{2}}{8\left(T_{4}-a_{4}\right)}+a_{3},
$$

where $J^{i j}$ are given by Eq. (16). Note that $T_{3}$ does not depend on $\omega^{5}$. On the hyperplane $T_{4}=T_{5}=0$ it reduces to

$$
-8 a_{4} T_{3}=\left(J^{A B}\right)^{2}-8 a_{3} a_{4}=0 .
$$

Eq. (24) states that the value of $S O(2,3)$-Casimir operator ${ }^{3}\left(J^{A B}\right)^{2}$ is equal to $8 a_{3} a_{4}$.

In the dynamical model constructed below, the equation $T_{3}=0$ appears as the first-class constraint. It implies that we are dealing with a theory with local symmetry, with the constraint being the generator of the symmetry [20]. The coordinate $\omega^{5}$ is not inert under the symmetry, $\delta \omega^{5} \sim\left\{T_{3}, \omega^{5}\right\}=\neq 0$. Hence $\omega^{5}$ is gauge non-invariant variable.

Summing up, we have restricted dynamics of spin on the surface (19), (20). If (21) are taken as coordinates of the phase space, the surface is the hyperplane $T_{4}=T_{5}=0$ with the coordinates $J^{5 \mu}, J^{0 i}, \omega^{5}$ subject to the condition (24). Since $\omega^{5}$ is gauge noninvariant coordinate, we can discard it.

This implies that we can quantize $J^{5 \mu}, J^{0 i}$ instead of the initial variables $\omega^{A}, \pi^{B}$. Similarly to the case for the $\Gamma$-matrices, the brackets of the variables $J^{5 \mu}, J^{0 i}$ do not form a closed Lie algebra. The nonclosed brackets are

$$
\left\{J^{5 i}, J^{5 j}\right\}=\left\{J^{0 i}, J^{0 j}\right\}=-2 J^{i j},
$$

where $J^{i j}=\left(J^{50}\right)^{-1}\left(J^{5 i} J^{0 j}-J^{5 j} J^{0 i}\right)$; see Eq. (17). Adding them to the initial variables, we obtain the set $J^{A B}=\left(J^{5 \mu}, J^{0 i}, J^{i j}\right)$ which obeys the desired algebra (13). According to Eqs. (9), (12), (13),

\footnotetext{
${ }^{3}$ In quantum theory, for the operators (12), 11) we have: $\hat{J}^{A B} \hat{J}_{A B}=20 \hbar^{2}$.
} 
quantization is achieved by replacing $J^{A B}$ on the $\Gamma$-matrices 4 . We assume that $\omega^{A}$ has the dimension of length; then $J^{A B}$ has the dimension of the Planck's constant. Hence the quantization rule is

$$
J^{5 \mu} \rightarrow \hbar \Gamma^{\mu}, \quad J^{\mu \nu} \rightarrow \hbar \Gamma^{\mu \nu} .
$$

This implies, that the Dirac equation (8) can be produced through the constraint

$$
T_{2} \equiv p_{\mu} J^{5 \mu}+m c \hbar=0 .
$$

Summing up, to describe the relativistic spin, we need a theory that implies the Dirac constraints (19), (20), (27) in the Hamiltonian formulation.

\section{Lagrangian action and the canonical quanti- zation}

One possible dynamical realization of the construction presented above is given by the following $d=4$ Poincaré-invariant Lagrangian

$$
\begin{array}{r}
L=-\frac{1}{2 e_{3}}\left[\left(\dot{x}^{\mu}+e_{2} \omega^{\mu}\right)^{2}-\left(e_{2} \omega^{5}\right)^{2}\right]-\frac{\sigma m c \hbar}{2 \omega^{5}}-\frac{\sigma^{2} a_{3}}{2 e_{3}}+ \\
\frac{1}{\sigma}\left[\left(\dot{x}^{\mu}+e_{2} \omega^{\mu}\right) \dot{\omega}_{\mu}-e_{2} \omega^{5} \dot{\omega}^{5}\right]-e_{4}\left(\omega^{A} \omega_{A}+a_{4}\right),
\end{array}
$$

written on the configuration space $x^{\mu}, \omega^{\mu}, \omega^{5}, e_{i}, \sigma$, where $e_{i}, \sigma$ are the auxiliary variables. The local symmetries of the theory are the reparametrizations as well as the following transformations with the parameter $\epsilon(\tau)$ (below we have defined $\beta \equiv \dot{e}_{4} \epsilon+\frac{1}{2} e_{4} \dot{\epsilon}$ )

$$
\begin{aligned}
& \delta x^{\mu}=0, \quad \delta \omega^{A}=\beta \omega^{A}, \quad \delta \sigma=\beta \sigma, \quad \delta e_{3}=0, \\
& \delta e_{2}=-\beta e_{3}+\frac{e_{2}}{\sigma} \dot{\beta}, \quad \delta e_{4}=-2 e_{4} \beta-\left(\frac{e_{2} \dot{\beta}}{2 \sigma^{2}}\right) .
\end{aligned}
$$

The presence of local symmetries implies the appearance of the firstclass constraints (19), (27) in the Hamiltonian formalism. We point out that in the Berezin-Marinov model the Dirac equation is implied by the supersymmetric gauge transformations. So, the symmetry (29) represents the bosonic analogue of these transformations.

\footnotetext{
${ }^{4}$ The matrices $\Gamma^{\mu}, \Gamma^{\mu \nu}$ are Hermitian operators with respect to the scalar product $\left(\Psi_{1}, \Psi_{2}\right)=\Psi_{1}^{\dagger} \Gamma^{0} \Psi_{2}$.
} 
Although there is no the constraint $p^{2}+m^{2} c^{2}=0$ in our model, our particle's speed cannot exceed the speed of light. Indeed, equations of motion for the auxiliary variables $e_{2}, e_{3}$ read $(\dot{x} \omega)=e_{2} a_{4}$, $\dot{x}^{2}+2 e_{2}(\dot{x} \omega)+e_{2}^{2} \omega^{A} \omega_{A}+\sigma^{2} a_{3}=0$. They imply $\left(\dot{x}^{\mu}\right)^{2}=-e_{2}^{2} a_{4}-\sigma^{2} a_{3}$. Since we are dealing with a reparametrization-invariant theory, we assume that the functions $x^{\mu}(\tau)$ represent the parametric equations of the trajectory $x^{i}(t)$. Then the previous equation implies

$$
\left(\frac{d x^{i}}{d t}\right)^{2}=\left(c \frac{\dot{x}^{i}(\tau)}{\dot{x}^{0}(\tau)}\right)^{2}=c^{2}\left(1-\frac{e_{2}^{2} a_{4}+\sigma^{2} a_{3}}{\left(\dot{x}^{0}\right)^{2}}\right)<c^{2},
$$

if we take $a_{3}>0, a_{4}>0$.

Curiously enough, the Lagrangian (28) can be rewritten in almost five-dimensional form. Namely, after the change $5\left(x^{\mu}, \sigma, e_{2}\right)$ $\rightarrow\left(\tilde{x}^{\mu}, \tilde{x}^{5}, \tilde{e}_{2}\right)$, where $\tilde{x}^{\mu}=x^{\mu}-\frac{e_{3}}{\sigma} \omega^{\mu}, \tilde{x}^{5}=-\frac{e_{3}}{\sigma} \omega^{5}, \tilde{e}_{2}=e_{2}+\left(\frac{e_{3}}{\sigma}\right)^{\cdot}$, it reads

$$
\begin{gathered}
L=-\frac{1}{2 e_{3}}\left(D \tilde{x}^{A}\right)^{2}+\frac{\left(\tilde{x}^{5}\right)^{2}}{2 e_{3}\left(\omega^{5}\right)^{2}}\left(\dot{\omega}^{A}\right)^{2}+\frac{e_{3} m c \hbar}{\tilde{x}^{5}}+\frac{e_{3} a_{3}\left(\omega^{5}\right)^{2}}{2\left(\tilde{x}^{5}\right)^{2}}- \\
e_{4}\left(\omega^{A} \omega_{A}+a_{4}\right),
\end{gathered}
$$

where we have defined $D \tilde{x}^{A}=\dot{\tilde{x}}^{A}+\tilde{e}_{2} \omega^{A}$.

Canonical quantization. To confirm that the action (28) leads to the Dirac equation, we construct its Hamiltonian formulation. In the Hamiltonian formalism, the equations (19), (20), (27) appear as the Hamiltonian constraints. The constraint $T_{2}$ has vanishing Poisson brackets with all the constraints. The remaining constraints obey the Poisson-bracket algebra

$$
\begin{gathered}
\left\{T_{3}, T_{4}\right\}=-4 T_{5}, \quad\left\{T_{3}, T_{5}\right\}=-2 T_{3}+2 a_{3}, \\
\left\{T_{4}, T_{5}\right\}=2 T_{4}-2 a_{4} .
\end{gathered}
$$

If we take the combination

$$
\tilde{T}_{3} \equiv T_{3}+\frac{a_{3}}{a_{4}} T_{4}
$$

the algebra acquires the form

$$
\left\{\tilde{T}_{3}, T_{4}\right\}=-4 T_{5}, \quad\left\{\tilde{T}_{3}, T_{5}\right\}=-2 T_{3}+2 \frac{a_{3}}{a_{4}} T_{4},
$$

\footnotetext{
${ }^{5}$ The change is an example of conversion of the second-class constraints in the Lagrangian formulation [18].
} 


$$
\left\{T_{4}, T_{5}\right\}=2 T_{4}-2 a_{4} .
$$

The only bracket which does not vanish on the constraint surface is $\left\{T_{4}, T_{5}\right\}$. According the Dirac terminology $[16,17]$, we have the first-class constraints (33), (27), and the second-class pair (20). The presence of the first-class constraints indicates that we are dealing with a theory invariant under a two-parameter group of local (gauge) symmetries, which has been discussed above.

The auxiliary variables $\sigma, e_{4}$ turn out to be subject to their own second-class constraints. Assuming that the constraints are taken into account by the transition from the Poisson to the Dirac bracket, the variables can be omitted from consideration [16, 17]. The Hamiltonian in terms of the remaining variables reads

$$
\begin{aligned}
& H=\frac{1}{2 e_{3}}\left(\frac{\omega^{5} e_{2}}{\pi^{5}}\right)^{2}\left(\pi^{A} \pi_{A}+a_{3}\right)+\frac{e_{2}}{2 \pi^{5}}\left(p_{\mu} J^{5 \mu}+m c \hbar\right)+ \\
& \lambda_{e 2} \pi_{e 2}+\lambda_{e 3} \pi_{e 3} \text {. }
\end{aligned}
$$

Here $\pi_{e a}$, are conjugate momenta for $e_{a}$ and $\lambda_{e a}$ are the Lagrangian multipliers for the primary constraints $\pi_{e a}=0$.

The constraints (19), (20) can also be taken into account by using of the Dirac bracket. Since they represent $S O(2,3)$-invariants, the Dirac brackets of the quantities $J^{A B}$ coincide with the Poisson one, Eq. (13). Hence we quantize the model according to Eq. (26). In the quantum theory, the first-class constraint (27) is imposed on the state vector. This gives the Dirac equation. In the result, canonical quantization of the model leads to the desired quantum picture.

\section{Solution to the classical equations of motion}

We now discuss some properties of the classical theory and confirm that they are in correspondence with those of the one-particle sector of the Dirac equation [19, 20].

Besides the constraints discussed above, the Hamiltonian (35) implies the following equations (we use the notation $(p \omega)=p^{\mu} \omega_{\mu}$ ):

$$
\begin{gathered}
\dot{e}_{a}=\lambda_{e a}, \quad \pi_{e a}=0, \quad a=2,3 ; \\
\dot{x}^{\mu}=\frac{e_{2}}{2 \pi^{5}} J^{5 \mu}, \quad \dot{p}^{\mu}=0 ;
\end{gathered}
$$




$$
\begin{array}{ll}
\dot{\omega}^{\mu}=\frac{1}{e_{3}}\left(\frac{e_{2} \omega^{5}}{\pi^{5}}\right)^{2} \pi^{\mu}+\frac{e_{2} \omega^{5}}{\pi^{5}} p^{\mu}, & \dot{\pi}^{\mu}=e_{2} p^{\mu}-2 e_{4} \omega^{\mu} \\
\dot{\omega}^{5}=\frac{1}{e_{3}}\left(\frac{e_{2} \omega^{5}}{\pi^{5}}\right)^{2} \pi^{5}+\frac{e_{2}}{\pi^{5}}(p \omega), & \dot{\pi}^{5}=\frac{e_{2}}{\pi^{5}}(p \pi)-2 e_{4} \omega^{5}
\end{array}
$$

Here $e_{4}=\frac{1}{2 e_{3}}\left(\frac{e_{2} \omega^{5}}{\pi^{5}}\right)^{2} \frac{a_{3}}{a_{4}}$. The equations reflect the fact that we are dealing with a theory with local symmetries. Indeed, we note that these equations do not determine the Lagrangian multipliers $\lambda_{e a}$, which enter as arbitrary functions into solutions to the equations of motion for $x^{\mu}, \omega^{A}, \pi^{A}$. According to the general theory $[16,17]$, variables with ambiguous dynamics do not represent the observable quantities. For our case, all the variables except $p_{\mu}$ turn out to be ambiguous.

To construct the gauge-invariant variables with unambiguous dynamics we first note that $x^{\mu}, p_{\mu}, J^{5 \mu}$ and $J^{\mu \nu}$ represent $\epsilon$-invariant quantities. Equations for the angular-momentum variables follow from (38)

$$
\dot{J}^{5 \mu}=-\frac{e_{2}}{\pi^{5}} J^{\mu \nu} p_{\nu}, \quad \dot{J}^{\mu \nu}=\frac{e_{2}}{\pi^{5}}\left(p^{\mu} J^{5 \nu}-p^{\nu} J^{5 \mu}\right) .
$$

In three-dimensional notation, these equations read

$$
\begin{gathered}
\dot{J}^{50}=-(\mathbf{W} \mathbf{p}), \quad \dot{\mathbf{J}}^{5}=-p^{0} \mathbf{W}+\mathbf{D} \times \mathbf{p}, \\
\dot{\mathbf{W}}=p^{0} \mathbf{J}^{5}-J^{50} \mathbf{p} .
\end{gathered}
$$

The ambiguity remaining in Eqs. (37), (39) due to the factor $\frac{e_{2}}{\pi^{5}}$ has a well-known interpretation, being related to the reparametrization invariance of the theory. We assume that, in accordance with this, the functions $x^{\mu}(\tau), J^{A B}(\tau)$ represent the dynamical variables $x^{i}(t)$, $J^{A B}(t)$ in the parametric form. Using the identity $\frac{d A(t)}{d t}=c \frac{\dot{A}(\tau)}{\dot{x}^{0}(\tau)}$, we obtain the deterministic evolution for the gauge-invariant variables:

$$
\begin{gathered}
\frac{d x^{i}}{d t}=c\left(J^{50}\right)^{-1} J^{5 i}, \quad \frac{d J^{5 \mu}}{d t}=-2 c\left(J^{50}\right)^{-1} J^{\mu \nu} p_{\nu}, \\
\frac{d J^{\mu \nu}}{d t}=2 c\left(J^{50}\right)^{-1}\left(p^{\mu} J^{5 \nu}-p^{\nu} J^{5 \mu}\right) .
\end{gathered}
$$

To find the trajectory $x^{i}(t)$, we take the equations (37), (39) in the gauge $\frac{e_{2}}{\pi^{5}}=1$ and note that they imply the following closed third-order equation for $x^{\mu}$

$$
\dddot{x}^{\mu}-p^{2} \dot{x}^{\mu}=\frac{m c \hbar}{2} p^{\mu} .
$$


For any $p^{2}<0$ its solution is given by

$$
x^{\mu}(\tau)=x_{0}^{\mu}-\frac{m c \hbar}{2 p^{2}} p^{\mu} \tau+\frac{a^{\mu}}{\sqrt{-p^{2}}} \cos \left(\sqrt{-p^{2}} \tau+\phi^{\mu}\right) .
$$

Since $x^{0}(\tau)$ in a reparametrization-invariant theory must be a monotonic function of $\tau$, we take the integration constant $a^{0}=0$. Then the parametric equations (43) imply

$$
x^{i}(t)=x_{0}^{i}+\frac{c p^{i}}{p_{0}} t+\frac{a^{i}}{\sqrt{-p^{2}}} \cos \left(\omega t+\phi^{i}\right) .
$$

The solution is a combination of the rectilinear motion and oscillations with the frequency

$$
\omega=\frac{2\left(-p^{2}\right)^{\frac{3}{2}}}{m \hbar p^{0}}
$$

For the particular value $p^{2}=-m^{2} c^{2}$, and when $p^{i} \ll p^{0}$, it approaches to the Compton frequency $\frac{2 m c^{2}}{\hbar}$. Hence the variable $x^{i}(t)$ experiences the Zitterbewegung in noninteracting theory.

As we have discussed above, our particle's speed cannot exceed the speed of light for any value of $p^{2}$. For the case $p^{2}>0$, the general solution to Eq. (42) represents hyperbolic motion

$$
x^{\mu}(\tau)=x_{0}^{\mu}-\frac{m c \hbar}{2 p^{2}} p^{\mu} \tau+\frac{a^{\mu}}{\sqrt{p^{2}}} \cosh \left(\sqrt{p^{2}} \tau+\phi^{\mu}\right) .
$$

The existence of such a self-accelerated solution for the Frenkel electron has been recently observed; see [21].

The variables free of Zitterbewegung. Besides the centre of charge, $\hat{x}$, in the Dirac theory we can construct the centre-of-mass (PryceNewton-Wigner) $[6,7]$ operator $\hat{\tilde{x}}$ in such a way that the conjugated momentum of $\hat{x}$ turns out to be the mechanical momentum for $\hat{\tilde{x}}$. So the Dirac particle looks like a kind of composed system (this picture has been used by Schrödinger [1] to identify spin with inner angular momentum of the system). The classical analogue of the centre-of-mass operator in our model is the variable

$$
\tilde{x}^{\mu}=x^{\mu}+\frac{1}{2 p^{2}} J^{\mu \nu} p_{\nu}
$$


It obeys the equation $\dot{\tilde{x}}^{\mu}(\tau)=-\frac{e_{2} m c \hbar}{2 \pi^{5} p^{2}} p^{\mu}$; then the centre of mass $\tilde{x}^{i}(t)$ moves along the straight line, $\frac{d \tilde{x}^{i}}{d t}=\frac{c p^{i}}{p^{0}}$. Note also that $p^{\mu}$ represents the mechanical momentum of the $\tilde{x}^{\mu}$-particle.

Let us take as the classical four-dimensional spin vector the PauliLubanski vector $S^{\mu}=\epsilon^{\mu \nu \alpha \beta} p_{\nu} J_{\alpha \beta}$. It has no precession in the free theory; $\dot{S}^{\mu}=0$. In the centre-of-charge instantaneous rest frame,

$$
J^{50}=\text { const }, \quad \mathbf{J}^{5}=0,
$$

it reduces to $S^{0}=0, \mathbf{S}=\mathbf{p} \times \mathbf{W}$. According to Eqs. (48), (18), only the part $\mathbf{W}$ of the angular-momentum tensor (16) survives in the nonrelativistic limit.

Comparison with the Barut-Zanghi (BZ) model. The BZ spinning particle [14] is widely used [22-27] for semiclassical analysis of spin effects. Starting from the even variables $z_{\alpha}$, where $\alpha=1,2,3,4$ is the $S O(1,3)$ spinor index, Barut and Zanghi have constructed an even spin tensor $S_{\mu \nu}=\frac{1}{4} i \bar{z} \gamma_{\mu \nu} z$. We point out that in the gauge $\frac{e_{2}}{\pi^{5}}=1$ our equations (37), (39) coincide with those of the BZ model, making the identifications $J^{5 \mu} \leftrightarrow v^{\mu}, J^{\mu \nu} \leftrightarrow S^{\mu \nu}$. Besides, our model implies the equations $\left(\frac{J^{5 \mu}}{\pi^{5}}\right)^{2}=-4 R, p_{\mu} J^{5 \mu}+m c \hbar=0$. The first equation guarantees that the centre of charge cannot exceed the speed of light. The second equation implies the Dirac equation 6 .

\section{Conclusion}

In this work we have constructed a semiclassical model (28), (35) which describes the one-particle sector of the Dirac equation. Although there is no the constraint $p^{2}+m^{2} c^{2}=0$ in our model, our particle's speed cannot exceed the speed of light. Spinning degrees of freedom are described on the basis of a seven-dimensional surface embedded in the ten-dimensional phase space $\omega^{A}, \pi^{A}$ equipped with the Poisson bracket (14). The surface is specified by the $S O(2,3)$ invariant equations (19), (20). The angular-momentum variables $J^{5 \mu}, J^{0 i}$ can be taken as coordinates of the surface. Quantizing them in accordance with their Poisson-bracket algebra (13), we have produced both the $\Gamma^{\mu}$-matrices and the relativistic spin tensor $J^{\mu \nu}$. The first-class constraint (27) is imposed on the state vectors, which leads to the Dirac equation.

${ }^{6}$ The Barut-Zanghi model [10] does not imply the Dirac equation. 
Our model shows the same undesirable properties as those of the Dirac equation in the semiclassical limit. We have solved the classical equations of motion and confirmed that the position variable $x^{i}$ experiences the Zitterbewegung in noninteracting theory, see (44). The variable $\tilde{x}^{\mu}$ specified by the equation (47) moves along a straight line and corresponds to the Pryce-Newton-Wigner operator of the Dirac theory. Like the Dirac equation, the model presented here gives no evidence as regards which of these two variables should be identified with the particle position.

We finish with a brief comment on a modification which solves the problems. We recall that the Dirac equation (8) implies the Klein-Gordon one. In contrast, in classical mechanics the corresponding constraint (27) does not imply the mass-shell constraint $p^{2}+m^{2} c^{2}=0$. So, the model presented here is not yet in complete correspondence with the Dirac theory. The semiclassical model that produces both constraints has been discussed in the recent work [28]. The extra first-class constraint implies that we are dealing with a theory with one more local symmetry, with the constraint being a generator of the symmetry $[29,17]$. This leads to a completely different picture of the classical dynamics. The variable $x^{\mu}$ is not inert under the extra symmetry; $\delta x^{\mu}=\beta p^{\mu}$, where $\beta(\tau)$ is the local parameter. Being gauge non-invariant, $x^{\mu}$ turns out to be an unobservable quantity. The variable $\tilde{x}^{\mu}$ of Eq. (47) is gauge invariant and should be identified with the position of the particle. Because $p^{\mu}$ is a mechanical momentum for $\tilde{x}^{\mu}$, the particle's speed cannot exceed the speed of light. In the absence of interaction it moves along the straight line. Hence the modified model is free of the undesirable Zitterbewegung.

\section{Acknowledgments}

This work was supported by the Brazilian foundation FAPEMIG.

\section{References}

[1] E. Schrödinger, Sitzunger. Preuss. Akad. Wiss. Phys.-Math. Kl. 24 (1930) 418. 
[2] A. H. Compton, J. of the Franklin Institute, 192 (1921) 145;

G. E. Uhlenbeck and S. Goudsmit, Nature, 117 (1926) 264.

[3] J. Frenkel, Z. fur Physik 37 (1926) 243.

[4] P. A. M. Dirac, Proc. Roy. Soc. A 117 (1928) 610; ibid. A 118 (1928) 351.

[5] M. H. L. Pryce, Proc. Roy. Soc. A 195 (1948) 62.

[6] T. D. Newton and E. P. Wigner, Rev. Mod. Phys 21 (1949) 400.

[7] L. L. Foldy and S. A. Wouthuysen, Phys. Rev. 78 (1950) 29.

[8] V. Bargmann, L. Michel and V.L. Telegdi, Phys. Rev. Lett. 2 (1959) 435.

[9] T. F. Jordan and N. Mukunda, Phys. Rev. 132 (1963) 1842.

[10] A. O. Barut Electrodynamics and Classical Theory of Fields and Particles, (MacMillan, New York 1964).

[11] F. A. Berezin and M. S. Marinov, JETP Lett. 21 (1975) 320; Ann. Phys. 104 (1977) 336.

[12] S. P. Gavrilov and D. M. Gitman, Int. J. Mod. Phys. A15, 4499 (2000).

[13] A. J. Hanson and T. Regge, Ann. Phys. 87 (1974) 498.

[14] A. O. Barut and N. Zanghi, Phys. Rev. Lett. 52 (1984) 2009.

[15] A. A. Deriglazov, Mod. Phys. Lett. A 25 (2010) 2769.

[16] D. M. Gitman and I. V. Tyutin, Quantization of Fields with Constraints (Springer-Verlag, Berlin, 1990).

[17] A. A. Deriglazov, Classical Mechanics, Hamiltonian and Lagrangian Formalism (Springer-Verlag, Berlin Heidelberg, 2010).

[18] A. A. Deriglazov, Z. Kuznetsova, Phys. Lett. B 646 (2007) 47.

[19] J. D. Bjorken, and S. D. Drell, Relativistic Quantum Fields (Hardcover Publisher: McGraw-Hill Companies, 1965).

[20] J. J. Sakurai, Modern Quantum Mechanics, Revised Edition (Addison-Wesley Publishing Company Inc., 1994), p. 68. 
[21] N. Kudryashova and Y. N. Obukhov, Phys. Lett. A 374 (2010) 3801.

[22] G. Salesi and E. Recami, Phys. Lett. A 267 (2000) 219.

[23] M. Pavsic, E. Recami, W. A. Rodrigues Jr., G. D. Maccarrone, F. Raciti and G. Salesi, Phys. Lett. B 318 (1993) 481.

[24] D. Hestenes, Found. Phys. 40 (2010) 1.

[25] D. Singh and N. Mobed, Class. Quantum Grav. 26 (2009) 185007.

[26] Nuri Unal, J. Math. Phys. 47 (2006) 092501.

[27] J. R. Van Meter, A. K. Kerman, P. Chen, and F. V. Hartemann, Phys. Rev. E 62 (2000) 8640.

[28] A. A. Deriglazov, Phys. Lett. A 376 (2012) 309; arXiv:1106.5228.

[29] A. A. Deriglazov and K. E. Evdokimov, Int. J. Mod. Phys. A 15 (2000) 4045; A. A. Deriglazov, J. Phys. A 40 (2007) 11083; J. Math. Phys. 50 (2009) 012907-1-15. 